\title{
Factors Associated with the Development of Postnatal Depression After Cesarean Delivery: A Prospective Study
}

This article was published in the following Dove Press journal: Neuropsychiatric Disease and Treatment

\author{
Carolyn Li-Jen Chan ${ }^{1, *}$ \\ Chin Wen Tan (iD) ${ }^{1,2, *}$ \\ Jason Ju In Chan' \\ Rehena Sultana ${ }^{3}$ \\ Tze-Ern Chua (iD) ${ }^{4,5}$ \\ Helen Yu Chen ${ }^{4,5}$ \\ Alex Tiong Heng Sia ${ }^{1,2}$ \\ Ban Leong Sng ${ }^{1,2}$ \\ 'Department of Women's Anesthesia, \\ KK Women's and Children's Hospital, \\ Singapore; ${ }^{2}$ Anesthesiology and \\ Perioperative Sciences Academic Clinical \\ Program, Duke-NUS Medical School, \\ Singapore; ${ }^{3}$ Centre for Quantitative \\ Medicine, Duke-NUS Medical School, \\ Singapore; ${ }^{4}$ Department of Psychological \\ Medicine, KK Women's and Children's \\ Hospital, Singapore; ${ }^{5}$ Pediatrics Academic \\ Clinical Program, Duke-NUS Medical \\ School, Singapore
}

*These authors contributed equally to this work
Correspondence: Ban Leong Sng

Tel +656394 I08I

Fax +65 6291266I

Email sng.ban.leong@singhealth.com.sg
Purpose: Pre-operative association factors (pain and psychological vulnerability) could significantly contribute to post-Cesarean pain; however, limited information is available on the development of postnatal depression (PND). We aimed to investigate the development of PND and its association with pain vulnerability and psychological vulnerability factors.

Patients and Methods: Women undergoing Cesarean delivery under spinal anesthesia were given pre-operative questionnaires, psychological and pain assessments including pain on local anesthetic injection during spinal anesthesia and mechanical temporal summation. Post-operative assessments were administered at 6 to 10 weeks post-Cesarean delivery via follow-up survey to assess post-Cesarean psychological and pain outcomes.

Results: PND occurred in 21.1\% (43 of 205) of patients who underwent elective Cesarean delivery. An increased pre-operative pain score with movement (Odds ratio (OR) 1.65, 95\% CI $1.12-2.44, \mathrm{p}=0.0110)$, anxiety about upcoming surgery (OR 1.02, 95\% CI 1.00-1.04, p = 0.0429), higher pre-operative Hospital Anxiety and Depression Scale (HADS) subscale on anxiety (OR 1.25, 95\% CI 1.07-1.45, p = 0.0041) and higher pre-operative central sensitization inventory (CSI) scores (OR 1.05, 95\% CI 1.01-1.09, p = 0.0156) were independently associated with an increased risk of PND. Anticipated pain medication needs were associated with reduced risk of PND (OR 0.48, 95\% CI 0.29-0.79, p = 0.0038) (Receiver operating characteristic $(\mathrm{ROC})=0.8177)$.

Conclusion: Higher pre-operative anxiety, pain score, central sensitization and lower anticipated pain medication needs were associated with increased risks of PND. Further work using larger sample size will be needed to validate the model in predicting PND development after Cesarean delivery.

Keywords: pain, central sensitization, anxiety, anticipated pain medication needs

\section{Introduction}

There is an increasing awareness of the importance of maternal mental health, with the worldwide prevalence of PND ranging from $7.6 \%$ to $39 \%$. $^{1,2}$ The effects of PND can be far reaching, as it can negatively impact not just on the health of the mother but also on her relationship with her baby, as well as subsequent infant physical and neurocognitive developmental outcomes. ${ }^{3}$ Additionally, PND may also contribute to chronic health conditions with associated social and economic consequences on families and society.

Cesarean delivery rates have been steadily increasing worldwide over the last few decades, contributing to an estimate of 29.7 million births in $2015 .^{4}$ While a potentially 
life-saving intervention when complications occur during childbirth, Cesarean delivery is considered as a major surgery that is accompanied by substantial tissue trauma and acute pain. Acute post-Cesarean pain, particularly if severe, is associated with persistent pain, which may negatively affect one's ability to care for one's newborn. ${ }^{5}$ Interestingly, a growing body of literature has reported the co-existence of persistent pain and depression, of which the risk of depression increases in the presence of worsening pain as assessed by severity, frequency and number of symptoms. ${ }^{6}$ Further studies demonstrated that up to $85 \%$ of those who suffered from persistent pain may acquire severe depression; ${ }^{6,7}$ and since the occurrence and development of persistent pain and depression are closely correlated, the comorbidities may further mutually promote the severities of respective conditions. ${ }^{8}$ Along with the variations in the use of different instruments or clinical interview for diagnosis, ${ }^{6}$ these undoubtedly heighten the challenges in treatments and outcome improving as compared with those with isolated morbidity.

Eisenach et al found that the severity of acute postnatal pain and an individual's response to pain, regardless of the mode of delivery and physical trauma, can predict PND. ${ }^{9}$ The mechanism for the genesis of depression, whether as an antecedent or consequence of persistent pain, is a subject of debate. $^{8}$ A number of studies have found an association between mode of delivery and the risk of developing PND. Cesarean delivery, in particular, has been found to carry higher risk for mothers' mental health compared with nonoperative deliveries. ${ }^{10-12}$ It was found that the responses to a pre-operative "three simple questions" (anxiety about upcoming surgery, anticipated pain score, anticipated pain medication needs) questionnaire could moderately predict the severity of acute post-Cesarean pain and the risk of persistent pain. ${ }^{13}$ Even though Cesarean delivery had been identified as a potential risk factor for PND, methodological weaknesses and the lack of randomization in trial design had led to difficulty in interpretation of the results. ${ }^{14}$

In this study, we aimed to investigate the pre-operative risk factors associated with the development of PND after Cesarean delivery and also investigated whether there is association between acute post-Cesarean pain with PND after Cesarean delivery.

\section{Materials and Methods}

\section{Patient Recruitment}

A prospective cohort study was conducted between May 2018 and April 2019 in KK Women's and
Children's Hospital, Singapore on females undergoing elective Cesarean delivery under neuraxial anesthesia. The study protocol was developed according to the Strengthening the Reporting of Observational studies in Epidemiology (STROBE) guidelines for reporting cohort studies. This study was reviewed and approved by the SingHealth Centralized Institutional Review Board, Singapore (SingHealth CIRB Ref: 2017/2381), and registered on Clinicaltrials.gov (NCT03645239).

Written informed consent was obtained from women scheduled for elective Cesarean deliveries in our institution, aged 21-50 years old, 36 gestational weeks or more, with an American Society of Anesthesiologists' (ASA) physical status of I and II, did not undergo the labor process and did not experience labor pain prior to the delivery. Patients with a previous history of persistent pain syndrome, intravenous drug or opioid abuse, as well as those undergoing emergency Cesarean delivery or Cesarean delivery under general anesthesia were excluded. Those who were unable to communicate in English were also excluded from the study, as standardized validated English questionnaires were administered among all ethnicities.

Pre-operative data were collected 1 to 2 hrs before Cesarean delivery and included patients' demographic data, history of morning sickness, history of smoking and pain scores (0-10; Numerical Rating Scale (NRS)) at rest or with movement (from supine to sitting) (Figure 1). The "three simple questions" were administered pre-operatively as below: ${ }^{13}$ i) Anxiety about upcoming surgery ("On a scale of $0-100$, with 0 being not anxious at all and 100 being extremely anxious, how anxious are you about your upcoming surgery?"); ii) Anticipated pain score (On a scale of $0-100$, with 0 being no pain at all and 100 most severe pain that you could imagine, how much pain do you anticipate experiencing after your upcoming surgery?); and iii) Anticipated pain medication needs ("On a scale of 0-5, with 0 being none at all, and 5 being much more than average, how much pain medication do you anticipate needing after your upcoming surgery?").

Mechanical temporal summation (MTS) was administered by using a 180-g von Frey filament applied to the patient's forearm before anesthesia. ${ }^{15}$ After one touch, the patient was asked to rate the pinprick pain score on a 0 100 verbal numerical pain scale. Ten consecutive (second to eleventh) touches were then applied within a $1 \mathrm{~cm}$ diameter circle at $1 \mathrm{~s}$ inter-stimulus intervals, and patient was later asked to rate the pain of the eleventh application. The differences between the eleventh and the first pinprick pain 


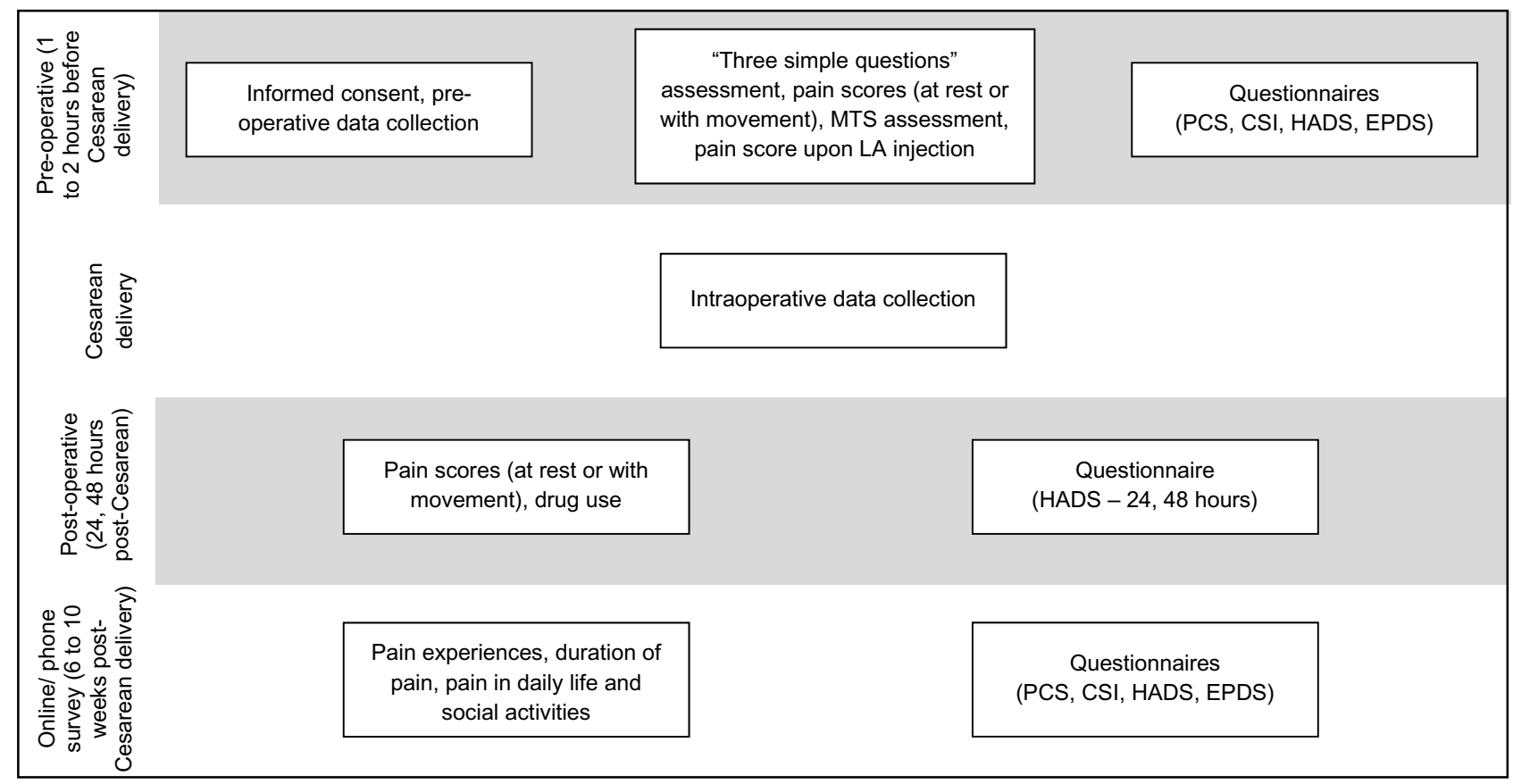

Figure I Study timeline.

Abbreviations: CSI, Central Sensitization Inventory; EPDS, Edinburgh Postnatal Depression Scale; HADS, Hospital Anxiety and Depression Scale; LA, local anesthetic; MTS, mechanical temporal summation; PCS, Pain Catastrophizing Scale.

scores were calculated, by which a positive difference $(>0)$ indicated the presence of an evoked MTS.

Pre-operative questionnaires were administered, including i) Pain Catastrophizing Scale (PCS), a validated psychometric instrument to evaluate the negative thought processes that patients may have when they are exposed to actual or anticipated pain or painful experiences; ${ }^{16}$ ii) Central Sensitization Inventory (CSI), an instrument to quantify patients' responses to expansion of the pain field and prolonged pain after stimulus removal; ${ }^{17}$ iii) Hospital Anxiety and Depression Scale (HADS) to assess the patients' level of anxiety and depression; ${ }^{18}$ and iv) Edinburgh Postnatal Depression Scale (EPDS), a self-reporting scale to evaluate PND. ${ }^{19}$

We also investigated the pain score upon local anesthetic (LA) injection prior to spinal anesthesia administration from each patient based on the 0-10 NRS immediately after the injection of $2-5 \mathrm{mls}$ of $1.0 \%$ lignocaine into the skin with a 22 Gauge hypodermic needle. To minimize the patient's subjective experience of pain, a standardized script was used to inform the patient right before the local anesthetic was injected. ${ }^{20}$ Intraoperative and postoperative anesthetic management was carried out at the attending clinician's discretion, based on routine standards of care. Spinal anesthesia comprising intrathecal hyperbaric bupivacaine $(10.5-12 \mathrm{mg})$, fentanyl $(15 \mathrm{mcg})$, and preservative-free morphine $(100 \mathrm{mcg})$ was administered for the Cesarean delivery.

Acute post-Cesarean pain, as defined by 24 and $48 \mathrm{hrs}$ post-Caesarean pain scores at rest and with movement were documented accordingly. Post-Cesarean analgesia and pain management were performed following the hospital protocols. Paracetamol and mefenamic acid were prescribed for regular oral administration and tramadol was prescribed when necessary until discharge. HADS questionnaires were completed $48 \mathrm{hrs}$ post-Cesarean delivery and before discharge from hospital to assess anxiety and depression scores during their ward stay.

An online survey was conducted at 6 to 10 weeks postCesarean delivery to investigate the primary outcome of PND using EPDS, and those who did not complete the online survey received phone calls to conduct the follow-up surveys. The surveys included pain experiences, duration of pain, and whether pain had affected their daily life and social activities. Surveys on pain vulnerability (PCS, CSI) and psychological vulnerability (HADS, EPDS) were also conducted.

\section{Sample Size Calculation and Statistical Analysis}

The calculated sample size of 190 (38 and 152) patients was based on the following assumptions: a conservative 
reference that higher acute post-Cesarean pain is associated with PND at 6 to 10 weeks post-Cesarean delivery $;^{9} 17 \%$ incidence of PND in Cesarean delivery based on Chandrasekaran et al; ${ }^{21} 20 \%$ severe acute postCesarean pain based upon Eisenach et al with an anticipated odds ratio of $3.2{ }^{9}$ 5\% type I error rate; $80 \%$ power and 1:4 allocation ratio. After adjusting for 11\% loss to follow-up, ineligibility and withdrawal, a sample size of 220 was required. In our study, the primary outcome of interest of PND was treated as binary data, with non-PND group having EPDS scoring of 0-9, and PND group with EPDS scoring of 10 and above. ${ }^{21}$

The categorical and continuous variables were summarized as frequency (proportion), mean \pm standard deviation (SD) or median [interquartile range (IQR)] based on the primary outcome of PND. Univariate and multivariate logistic regression analyses were done to examine the primary outcome of finding the associated factors. The association of any independent variable from logistic regression model was expressed as odds ratio (OR) with the corresponding 95\% confidence interval (95\% CI). Multivariate logistic regression was performed using variables with $\mathrm{p}<0.05$ in the univariate analysis. Stepwise variable selection method was used to identify significant explanatory variables. We also looked at the area under the curve from receiver operating characteristics (ROC) curve based on the generated multivariate model. Significance level was set at 0.05 and all tests were two-tailed. SAS version 9.4 software (SAS Institute; Cary, North Carolina, USA) was used for all analyses.

\section{Results}

We recruited 220 women in this study, with two patients excluded (less than 36 gestational weeks, undergoing general anesthesia (Figure 2). Further 13 patients were excluded from analysis due to loss to follow-up, withdrawal prior to the 6 to 10 weeks post-Cesarean surveys, or incomplete EPDS survey. At 6 to 10 weeks post-Cesarean delivery, 205 patients were categorized into two groups, with the "non-PND" group having EPDS score of less than 10 at 6 to 10 weeks post-Cesarean delivery $(n=162)$, and the "PND" group having EPDS score of equal to or more than $10(n=43)$. In this study, the overall prevalence of PND at 6 to 10 weeks post-Cesarean delivery was found to be $21.1 \%$ (95\% CI 15.6-26.2\%). The demographic characteristics of both groups are shown in Table 1.

We examined the association of pain measures with PND at 6 to 10 weeks post-Cesarean delivery (Table 2). There was a significant association found between increased preoperative pain score with movement (OR 1.49, 95\% CI 1.09-2.04, p $=0.0120$ ) and PND at 6 to 10 weeks postCesarean delivery, but pre-operative pain score at rest was not an association factor. One patient received for additional nitrous oxide, and three patients were administered intravenous ketamine (range $10-50 \mathrm{mg}$ ) during the intraoperative period. There was no significant difference in the $24 \mathrm{hrs}$ and $48 \mathrm{hrs}$ post-Cesarean pain scores between the PND and nonPND groups. Of the "three simple questions" conducted preoperatively, in relation to the secondary outcomes of this study, a greater anxiety about upcoming surgery was significantly associated with PND at 6 to 10 weeks post-Cesarean delivery (OR 1.02, 95\% CI 1.01-1.04, p = 0.0112); and a greater anticipated pain medication needs were associated with lower risk of PND (OR 0.65, 95\% CI 0.44-0.95, p = 0.0274). Both the evoked MTS (difference between the eleventh and the first pinprick pain scores $>0$ ) and the anticipated pain score was not significantly associated with PND at 6 to 10 weeks post-Cesarean delivery.

We further performed chi-squared tests to determine whether greater anticipated pain medication needs $(\geq 3)$ was associated with the 24 and 48 hrs post-Cesarean pain scores. By stratifying the patients into no or mild pain (pain scores $0-3$ ) and moderate to severe pain (pain scores 4-10), we found that there was no significant association between greater anticipated pain medication needs $(\geq 3)$ and $24 \mathrm{hrs}$ (at rest, $\mathrm{p}=0.6227$; with movement, $\mathrm{p}=0.2606$ ) and $48 \mathrm{hrs}$ (at rest, $\mathrm{p}=0.6569$; with movement, $\mathrm{p}=0.1432$ ) postCesarean pain scores. There was also no association between greater anticipated pain medication needs $(\geq 3)$ with presence of past Cesarean delivery $(p=0.3861)$ or number of children $(p=0.7397)$. From the follow-up survey, no significant difference was found between PND and non-PND groups on the presence of pain around their scar or abdominal wound site for 4 weeks or more $(p=0.4090)$.

We also investigated the psychological and pain-related measures in PND and non-PND groups (preoperatively (EPDS, HADS, CSI, and PCS), during ward stay (HADS) and 6 to 10 weeks post-Cesarean delivery (EPDS, HADS, CSI, and PCS; Table 3). In the non-PND group, the mean \pm SD pre-operative EPDS score was $6.1 \pm 3.6$ and further decreased to $4.3 \pm 2.8$ at 6 to 10 weeks post-Cesarean delivery. In contrast, PND group had a higher mean \pm SD EPDS score $(13.3 \pm 3.8)$ at 6 to 10 weeks post-Cesarean delivery as compared with pre-operative EPDS scores $(9.9 \pm$ 4.0). With regard to PCS questionnaire, higher pre-operative pain rumination scores (OR 1.09, 95\% CI 1.00-1.18, p = 


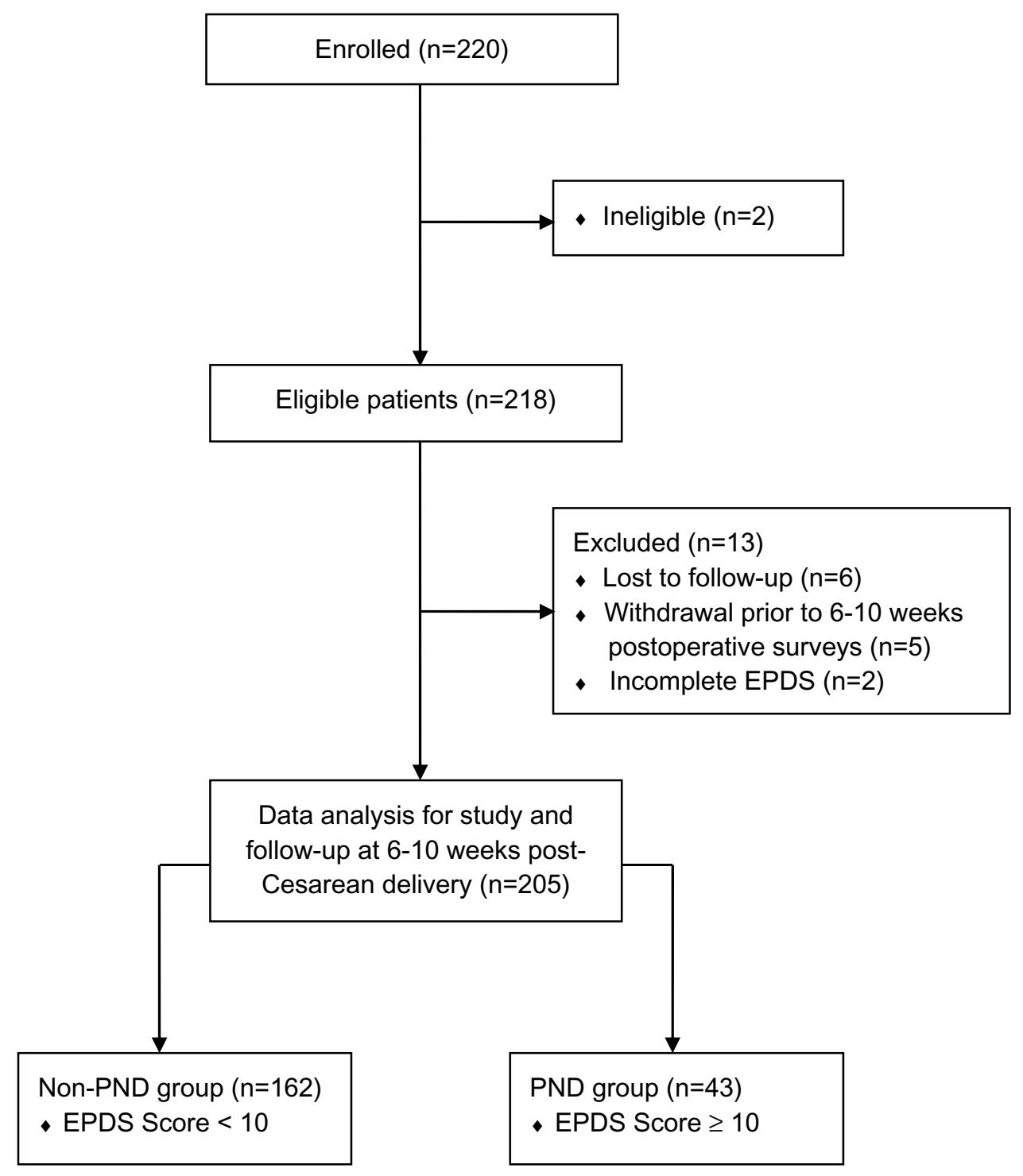

Figure 2 Study flowchart.

Abbreviations: EPDS, Edinburgh Postnatal Depression Scale; PND, postnatal depression.

0.0442), magnification scores (OR 1.17, 95\% CI 1.03-1.34, $\mathrm{p}=0.0188)$ and total scores (OR 1.04, 95\% CI 1.00-1.07, $\mathrm{p}=$ 0.0370 ) were associated with PND at 6 to 10 weeks postCesarean delivery (Table 3 ). An increased pre-operative CSI (OR 1.06, 95\% CI 1.03-1.09, $\mathrm{p}=0.0006$ ), HADS (depression score: OR 1.20, 95\% CI 1.08-1.34, $\mathrm{p}=0.001$; anxiety score: OR 1.3, 95\% CI 1.16-1.46, p<0.0001) and EPDS (OR $1.31,95 \%$ CI $1.18-1.45, \mathrm{p}<0.0001)$ also showed significant association with a higher risk of PND at 6 to 10 weeks postCesarean delivery. HADS scores at $48 \mathrm{hrs}$ post-Cesarean delivery for both depression scores (OR 1.11, 95\% CI $1.03-1.20, \mathrm{p}=0.0096)$ and anxiety scores (OR 1.22, 95\% CI 1.11-1.33, $\mathrm{p}<0.0001)$ were also significantly associated with PND at 6 to 10 weeks post-Cesarean delivery. At 6 to 10 weeks post-Cesarean delivery, increased HADS, PCS and CSI showed significant association with PND at 6 to 10 weeks post-Cesarean delivery.

Univariate logistic regression analysis showed that patients with existing children, and previous Cesarean delivery were associated with a lower risk of PND, whereas a higher score in pre-operative pain with movement, anxiety about upcoming surgery, anticipated pain medication needs, pre-operative PCS (rumination, magnification, total), pre-operative CSI and HADS (depression, anxiety) were associated with a higher risk of development of PND. These univariate factors $(p<0.05)$ were considered for the multivariate logistic regression (Table 4). Five independent association factors for PND were identified: Increased pre-operative pain score (with 
Table I Demographic Characteristics and Univariate Analyses (Based on EPDS Score at 6 to 10 Weeks Post-Cesarean Survey)

\begin{tabular}{|c|c|c|c|c|}
\hline Characteristics & $\begin{array}{l}\text { Non-PND Group (EPDS } \\
\text { Score }<10) N=162\end{array}$ & $\begin{array}{l}\text { PND Group (EPDS } \\
\text { Score } \geq 10) \quad N=43\end{array}$ & Unadjusted OR $(95 \% \mathrm{CI})$ & P-value \\
\hline Age (years) & $34.9 \pm 5.2$ & $34.1 \pm 4.4$ & $0.97(0.90,1.04)$ & 0.3692 \\
\hline $\begin{array}{l}\text { Race } \\
\text { Chinese } \\
\text { Malay } \\
\text { Indian } \\
\text { Others }\end{array}$ & $\begin{array}{l}96(59.3) \\
39(24.1) \\
13(8.0) \\
14(8.6)\end{array}$ & $\begin{array}{l}28(65.1) \\
4(9.3) \\
7(16.3) \\
4(9.3)\end{array}$ & $\begin{array}{l}\text { I } \\
0.35(0.12,1.07) \\
1.85(0.67,5.07) \\
1.00(0.30,3.21)\end{array}$ & $\begin{array}{l}0.1306^{\mathrm{a}} \\
- \\
0.0333 \\
0.0728 \\
0.8418\end{array}$ \\
\hline $\begin{array}{l}\text { Weight }(\mathrm{kg}) \\
\text { Height }(\mathrm{cm}) \\
\text { BMI }\left(\mathrm{kg} / \mathrm{m}^{2}\right)\end{array}$ & $\begin{array}{l}73.2 \pm 12.4 \\
159.0 \pm 5.7 \\
29.0 \pm 4.5\end{array}$ & $\begin{array}{l}71.9 \pm 13.2 \\
159.4 \pm 6.0 \\
28.2 \pm 4.5\end{array}$ & $\begin{array}{l}0.99(0.96,1.02) \\
1.01(0.96,1.07) \\
0.96(0.89,1.04)\end{array}$ & $\begin{array}{l}0.5336 \\
0.6605 \\
0.3419\end{array}$ \\
\hline $\begin{array}{l}\text { ASA physical status } \\
\text { I } \\
\text { II }\end{array}$ & $\begin{array}{l}101(62.3) \\
61(37.7)\end{array}$ & $\begin{array}{l}25(58.1) \\
18(41.9)\end{array}$ & $1.19(0.60,2.36)$ & $\begin{array}{l}- \\
0.6146\end{array}$ \\
\hline $\begin{array}{l}\text { Children } \\
\text { No } \\
\text { Yes }\end{array}$ & $\begin{array}{l}40(24.7) \\
122(75.3)\end{array}$ & $\begin{array}{l}19(44.2) \\
24(55.8)\end{array}$ & $\begin{array}{l}\text { I } \\
0.4 \mathrm{I}(0.2 \mathrm{I}, 0.83)\end{array}$ & $\begin{array}{l}- \\
0.0136\end{array}$ \\
\hline $\begin{array}{l}\text { Number of Children } \\
\text { Gestational age (weeks) }\end{array}$ & $\begin{array}{l}1.6 \pm 0.8 \\
38.4 \pm 0.8\end{array}$ & $\begin{array}{l}1.5 \pm 1.0 \\
38.6 \pm 0.5\end{array}$ & $\begin{array}{l}0.92(0.53,1.60) \\
1.42(0.66,3.07)\end{array}$ & $\begin{array}{l}0.7542 \\
0.3918\end{array}$ \\
\hline $\begin{array}{l}\text { Smoking } \\
\text { No } \\
\text { Yes }\end{array}$ & $\begin{array}{l}156(96.3) \\
6(3.7)\end{array}$ & $\begin{array}{l}42(97.7) \\
\mathrm{I}(2.3)\end{array}$ & $\begin{array}{l}\text { I } \\
0.62(0.07,5.28)\end{array}$ & $\begin{array}{l}- \\
0.6611\end{array}$ \\
\hline $\begin{array}{l}\text { Exercise } \\
\text { No } \\
\text { Yes }\end{array}$ & $\begin{array}{l}113(69.8) \\
49(30.2)\end{array}$ & $\begin{array}{l}26(60.5) \\
17(39.5)\end{array}$ & $1.51(0.75,3.03)$ & $\begin{array}{l}- \\
0.2482\end{array}$ \\
\hline $\begin{array}{l}\text { Presence of comorbidities } \\
\text { No } \\
\text { Yes }\end{array}$ & $\begin{array}{l}109(67.3) \\
53(32.7)\end{array}$ & $\begin{array}{l}24(55.8) \\
19(44.2)\end{array}$ & $1.63(0.82,3.23)$ & $\begin{array}{l}- \\
0.1633\end{array}$ \\
\hline $\begin{array}{l}\text { Past Cesarean delivery } \\
\text { No } \\
\text { Yes }\end{array}$ & $\begin{array}{l}49(30.2) \\
113(69.8)\end{array}$ & $\begin{array}{l}21(48.8) \\
22(51.2)\end{array}$ & $0.45(0.23,0.90)$ & $\begin{array}{l}- \\
0.0241\end{array}$ \\
\hline
\end{tabular}

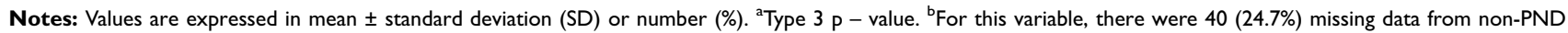
group with EPDS score <10, and 19 (44.2\%) missing data from PND group with EPDS score $\geq 10$ at 6 to 10 weeks post-Cesarean survey. ${ }^{\mathrm{C}}$ The comorbidities assessed included gestational diabetes mellitus $(n=32)$, high BMI $(n=17)$, history of past/current asthma $(n=12)$, hypertension $(n=5)$, hypothyroidism $(n=4)$ etc. Patients were taking non-pain related medications, with the majority being metformin $(n=4)$, thyroxine $(n=3)$, nifedipine $(n=3)$, insulin $(n=2)$, symbicort $(n=2)$ and methyldopa $(n=2)$.

Abbreviations: ASA, American Society of Anesthesiologists; BMI, body mass index; Cl, confidence interval; EPDS, Edinburgh Postnatal Depression Scale; OR, odds ratio; PND, postnatal depression.

movement; adjusted OR 1.65, 95\% CI 1.12-2.44, $\mathrm{p}=0.0110)$, increased anxiety about upcoming surgery (adjusted OR 1.02, 95\% CI 1.00-1.04, $\mathrm{p}=0.0429$ ), decreased anticipated pain medication needs (adjusted OR 0.48, 95\% CI 0.29-0.79, p = 0.003 ), increased pre-operative CSI (adjusted OR 1.05, 95\% CI 1.01-1.09, $\mathrm{p}=0.0156$ ) and increased pre-operative HADS subscale on anxiety (adjusted OR 1.25, 95\% CI 1.07-1.45, $\mathrm{p}=$ $0.0041)$. The area under curve of ROC of this multivariate model was 0.8177 (Figure 3).

\section{Discussion}

In this Asian population cohort study, increased preoperative pain score with movement, anxiety about upcoming surgery, overall anxiety as measured by pre-operative HADS, and pre-operative central sensitization were significantly associated with an increased risk of PND at 6 to 10 weeks post-Cesarean delivery. Interestingly, greater anticipated pain medication needs were associated with decreased risk of PND at 6 to 10 weeks post-Cesarean delivery. 
Table 2 Pain Characteristics and Univariate Analyses (Based on EPDS Score at 6 to 10 Weeks Post-Cesarean Survey)

\begin{tabular}{|c|c|c|c|c|}
\hline Characteristics & $\begin{array}{l}\text { Non-PND Group (EPDS } \\
\text { Score }<10) N=162\end{array}$ & $\begin{array}{l}\text { PND Group (EPDS } \\
\text { Score } \geq 10) N=43\end{array}$ & $\begin{array}{l}\text { Unadjusted OR } \\
(95 \% \mathrm{CI})\end{array}$ & P-value \\
\hline \multicolumn{5}{|l|}{ Pre-operative } \\
\hline Pain score (at rest; 0-10) & $1.7 \pm 2.0$ & $2.0 \pm 2.6$ & $1.38(0.99,1.93)$ & 0.0581 \\
\hline Pain score (with movement; 0-10) & $4.4 \pm 2.4$ & $4.8 \pm 2.8$ & $1.49(1.09,2.04)$ & 0.0120 \\
\hline Anxiety about upcoming surgery $(0-100)$ & $50.0[40.0]$ & $65.0[30.0]$ & $1.02(1.01,1.04)$ & 0.0112 \\
\hline Anticipated pain score $(0-100)$ & $70.0[20.0]$ & $80.0[30.0]$ & $0.99(0.98,1.01)$ & 0.3774 \\
\hline Anticipated pain medication needs $(0-5)$ & $3.0 \pm 0.7$ & $2.7 \pm 1.1$ & $0.65(0.44,0.95)$ & 0.0274 \\
\hline $\begin{array}{l}\text { Difference between eleventh and first pinprick } \\
\text { pain scores }\end{array}$ & $-9.3 \pm 14.8$ & $-7.9 \pm 14.4$ & $1.00(0.98,1.03)$ & 0.5620 \\
\hline \multicolumn{5}{|l|}{ Presence of evoked MTS } \\
\hline No & $54(33.5)$ & $12(27.9)$ & I & - \\
\hline Yes & $107(66.5)$ & $31(72.1)$ & $1.30(0.62,2.74)$ & 0.4838 \\
\hline Pain score on LA injection (0-10) & $4.7 \pm 2.2$ & $4.7 \pm 2.3$ & $1.00(0.86,1.17)$ & 0.9697 \\
\hline \multicolumn{5}{|l|}{ Wound infiltration with LA at the end of surgery } \\
\hline No & $161(99.4)$ & $42(97.7)$ & 1 & - \\
\hline Yes & $\mathrm{I}(0.6)$ & I (2.3) & $3.83(0.24,62.57)$ & 0.3456 \\
\hline \multicolumn{5}{|l|}{ Intraoperative morphine used } \\
\hline No & $\mathrm{I}(0.6)$ & I (2.3) & I & - \\
\hline Yes & $161(99.4)$ & $42(97.7)$ & $0.26(0.02,4.26)$ & 0.3456 \\
\hline Dose of morphine if used (mg) & $0.1 \pm 0.0$ & $0.1 \pm 0.0$ & - & - \\
\hline \multicolumn{5}{|l|}{ Intraoperative fentanyl used } \\
\hline No & $\mathrm{I}(0.6)$ & I (2.3) & 1 & - \\
\hline Yes & I6I (99.4) & $42(97.7)$ & $0.26(0.02,4.26)$ & 0.3456 \\
\hline Dose of fentanyl if used (mcg) & $16.2 \pm 8.9$ & $22.5 \pm 24.6$ & $1.03(1.00,1.05)$ & 0.0315 \\
\hline \multicolumn{5}{|l|}{ Intraoperative paracetamol used } \\
\hline No & $122(75.3)$ & $23(53.5)$ & 1 & - \\
\hline Yes & $40(24.7)$ & $20(46.5)$ & $2.65(1.32,5.33)$ & 0.0061 \\
\hline Dose of paracetamol if used (mg) & $1300.0 \pm 0.0$ & $1270.0 \pm 92.3$ & $0.95(0.02,43.60)$ & 0.9806 \\
\hline \multicolumn{5}{|l|}{ Post-operative (24 hrs) } \\
\hline Pain score (at rest; $0-10$ ) & $1.7 \pm 2.0$ & $2.0 \pm 2.6$ & $1.09(0.93,1.27)$ & 0.2792 \\
\hline Pain score (with movement; 0-I0) & $4.4 \pm 2.4$ & $4.8 \pm 2.8$ & $1.07(0.93,1.23)$ & 0.3574 \\
\hline \multicolumn{5}{|l|}{ Paracetamol Used } \\
\hline No & $6(3.7)$ & $3(7.0)$ & 1 & - \\
\hline Yes & $156(96.3)$ & $40(93.0)$ & $0.5 \mathrm{I}(0.12,2.14)$ & 0.3596 \\
\hline Dose of Paracetamol if used (mg) & $2189.0 \pm 777.4$ & $2125.0 \pm 723.0$ & $1.00(0.999,1.000)$ & 0.6616 \\
\hline \multicolumn{5}{|l|}{ Mefenamic Acid Used } \\
\hline No & $35(2 \mid .6)$ & $6(14.0)$ & 1 & - \\
\hline Yes & I 27 (78.4) & $37(86.0)$ & $1.70(0.66,4.35)$ & 0.2690 \\
\hline Dose of Mefenamic Acid if used (mg) & $953.5 \pm 261.0$ & $932.0 \pm 268.0$ & $1.00(0.998,1.001)$ & 0.6675 \\
\hline \multicolumn{5}{|l|}{ Tramadol Used } \\
\hline No & $15 \mid(93.2)$ & $43(100)$ & 1 & - \\
\hline Yes & II (6.8) & $0(0)$ & $0.15(0.01,2.97)$ & 0.2139 \\
\hline
\end{tabular}


Table 2 (Continued).

\begin{tabular}{|c|c|c|c|c|}
\hline Characteristics & $\begin{array}{l}\text { Non-PND Group (EPDS } \\
\text { Score }<10) N=162\end{array}$ & $\begin{array}{l}\text { PND Group (EPDS } \\
\text { Score } \geq 10) N=43\end{array}$ & $\begin{array}{l}\text { Unadjusted OR } \\
(95 \% \mathrm{CI})\end{array}$ & P-value \\
\hline Dose of Tramadol if used (mg) & $77.3 \pm 26.1$ & - & - & - \\
\hline \multicolumn{5}{|l|}{ Post-operative (48 hrs) } \\
\hline Pain score (at rest; $0-10$ ) & $1.7 \pm 2.0$ & $1.8 \pm 2.2$ & $\mathrm{I} .03(0.87, \mathrm{I} .2 \mathrm{I})$ & 0.7569 \\
\hline Pain score (with movement; 0-10) & $4.8 \pm 2.0$ & $5.0 \pm 2.4$ & $1.05(0.89,1.24)$ & 0.5401 \\
\hline \multicolumn{5}{|l|}{ Paracetamol Used } \\
\hline No & $7(4.3)$ & $2(4.7)$ & 1 & - \\
\hline Yes & $155(95.7)$ & $4 \mid(95.3)$ & $0.93(0.19,4.63)$ & 0.9252 \\
\hline Dose of Paracetamol if used (mg) & $3164.0 \pm 760.5$ & $2865.9 \pm 766.7$ & $0.94(0.90,0.99)$ & 0.0223 \\
\hline \multicolumn{5}{|l|}{ Mefenamic Acid Used } \\
\hline No & $31(19.1)$ & $4(9.3)$ & I & - \\
\hline Yes & |3| (80.9) & $39(90.7)$ & $2.31(0.77,6.94)$ & 0.1367 \\
\hline Dose of Mefenamic Acid if used (mg) & $1475.4 \pm 596.9$ & $1270.0 \pm 408.7$ & $0.99(0.66, I .48)$ & 0.9496 \\
\hline \multicolumn{5}{|l|}{ Tramadol Used } \\
\hline No & I 42 (87.7) & $40(93.0)$ & 1 & - \\
\hline Yes & $20(12.3)$ & $3(7.0)$ & $0.53(0.15,1.88)$ & 0.3282 \\
\hline Dose of Tramadol if used (mg) & $98.8 \pm 56.5$ & $66.7 \pm 28.9$ & $0.86(0.10,7.68)$ & 0.8950 \\
\hline \multicolumn{5}{|l|}{ Post-operative (6 to 10 weeks) } \\
\hline \multicolumn{5}{|c|}{$\begin{array}{l}\text { Presence of pain around their scar or abdominal } \\
\text { wound site for } 4 \text { weeks or more }\end{array}$} \\
\hline No & $140(86.4)$ & $35(81.4)$ & 1 & - \\
\hline Yes & $22(13.6)$ & $8(18.6)$ & $1.46(0.60,3.54)$ & 0.4090 \\
\hline
\end{tabular}

Notes: Values are expressed in mean \pm standard deviation (SD), median [interquartile range (IQR)] or number (\%).

Abbreviations: $\mathrm{Cl}$, confidence interval; EPDS, Edinburgh Postnatal Depression Scale; LA, local anesthetic; MTS, mechanical temporal summation; OR, odds ratio; PND, postnatal depression.

Cesarean delivery involves major tissue injury and is considered as major surgery with its associated significant postsurgical pain. Previous studies suggest reasons such as existing pain problems, tocophobia (fear of childbirth), concerns on incontinence and pelvic organ prolapse, and desire to avoid later maternal and neonate morbidities could all contribute to patients requesting for elective Cesarean delivery. ${ }^{22}$ Additionally, Eisenach et al reported that severe acute post-Cesarean pain is an independent predictor of PND. However, in this study higher 24 and 48 hrs postCesarean pain scores were not associated with a higher risk of PND. ${ }^{9}$ We also found some patients were having pain prior to their Cesarean delivery, which may need further investigation on its implication to pain development. Eisenach et al had used a more stringent EPDS cut-off of $\geq 13$ to determine occurrence of PND; and in this study, we evaluated the pain scores at rest and with movement.
Hence, different definitions and categorization of factors used could have contributed to the difference in findings. Pain existing before the Cesarean delivery, a factor usually not enquired prior to elective Cesarean deliveries, is an important association of PND. Interestingly, pre-operative EPDS scores was not found to be an independent association factor in this study's model, although previous studies have found higher prevalence rates of depression during pregnancy than postnatal period. ${ }^{23,24}$ Our finding suggests the importance of looking at other pain related and psychological related factors, on top of pre-delivery EPDS score assessment for patients undergoing Cesarean delivery.

Pan et al previously showed that the use of "three simple questions" could aid in identifying those with evoked pain score with movement following the first day post-Cesarean delivery. Based on the fact that the severity of acute post-Cesarean pain could predict the PND 
Table 3 Psychological Characteristics and Univariate Analyses (Based on EPDS Score at 6 to 10 Weeks Post-Cesarean Survey)

\begin{tabular}{|c|c|c|c|c|}
\hline Parameters & $\begin{array}{l}\text { Non-PND Group (EPDS } \\
\text { Score }<10) N=162\end{array}$ & $\begin{array}{l}\text { PND Group (EPDS } \\
\text { Score } \geq 10) N=43\end{array}$ & $\begin{array}{l}\text { Unadjusted OR } \\
(95 \% \mathrm{CI})\end{array}$ & p-value \\
\hline \multicolumn{5}{|l|}{ Pre-operative } \\
\hline PCS- Rumination (0-16) & $5.2 \pm 4.1$ & $6.7 \pm 4.1$ & $1.09(1.00,1.18)$ & 0.0442 \\
\hline PCS- Magnification (0-12) & $3.0 \pm 2.4$ & $4.0 \pm 3.0$ & $1.17(1.03,1.34)$ & 0.0188 \\
\hline PCS- Helplessness (0-24) & $5.4 \pm 4.5$ & $6.6 \pm 4.8$ & $1.06(0.99,1.13)$ & 0.1211 \\
\hline PCS- Total Score (0-52) & $13.6 \pm 10.0$ & $17.3 \pm 10.5$ & $1.04(1.00,1.07)$ & 0.0370 \\
\hline CSI $(0-100)$ & $52.0 \pm 11.0$ & $59.2 \pm 12.1$ & $1.06(1.03,1.09)$ & 0.0006 \\
\hline HADS - Depression (0-2I) & $3.8 \pm 2.8$ & $5.6 \pm 3.4$ & $1.20(1.08,1.34)$ & 0.0010 \\
\hline HADS - Anxiety (0-2I) & $6.1 \pm 3.0$ & $8.8 \pm 3.4$ & $1.30(1.16,1.46)$ & $<0.0001$ \\
\hline HADS- Total Score (0-42) & $9.9 \pm 5.06$ & $14.4 \pm 6.04$ & $1.16(1.09,1.24)$ & $<0.0001$ \\
\hline EPDS $(0-30)$ & $6.1 \pm 3.6$ & $9.9 \pm 4.0$ & $1.31(1.18,1.45)$ & $<0.0001$ \\
\hline \multicolumn{5}{|l|}{ Post-operative (48 hrs) } \\
\hline HADS - Depression (0-2I) & $5.0 \pm 4.1$ & $6.9 \pm 3.6$ & I.II $(1.03,1.20)$ & 0.0096 \\
\hline HADS - Anxiety (0-2I) & $5.7 \pm 3.6$ & $8.6 \pm 4.2$ & $1.22(1.11,1.33)$ & $<0.0001$ \\
\hline HADS- Total Score (0-42) & $10.7 \pm 6.9$ & $15.5 \pm 7 . \mid$ & $1.09(1.04,1.15)$ & 0.0002 \\
\hline \multicolumn{5}{|l|}{ Post-operative (6 to 10 weeks) } \\
\hline PCS - Rumination (0-16) & $2.7 \pm 3.3$ & $4.3 \pm 3.5$ & $1.14(1.04,1.25)$ & 0.0062 \\
\hline PCS- Magnification (0-I2) & $1.5 \pm 1.8$ & $2.7 \pm 2.1$ & $1.32(1.12,1.56)$ & 0.0010 \\
\hline PCS- Helplessness (0-24) & $2.7 \pm 3.4$ & $4.9 \pm 3.7$ & $1.17(1.06,1.28)$ & 0.0010 \\
\hline PCS- Total Score (0-52) & $6.9 \pm 7.9$ & $11.9 \pm 8.6$ & $1.07(1.03,1.11)$ & 0.0011 \\
\hline CSI $(0-100)$ & $44.7 \pm 10.6$ & $58.7 \pm 10.6$ & $1.13(1.09,1.18)$ & $<0.0001$ \\
\hline HADS - Depression (0-2I) & $2.9 \pm 2.7$ & $7.4 \pm 3.3$ & $1.54(1.35,1.76)$ & $<0.0001$ \\
\hline HADS - Anxiety (0-2I) & $2.9 \pm 2.2$ & $8.2 \pm 3.1$ & $2.21(1.74,2.80)$ & $<0.0001$ \\
\hline HADS- Total Score (0-42) & $5.8 \pm 4.0$ & $15.6 \pm 5.7$ & $1.48(1.32,1.66)$ & $<0.0001$ \\
\hline EPDS $(0-30)$ & $4.3 \pm 2.8$ & $13.3 \pm 3.8$ & 1 & - \\
\hline
\end{tabular}

Notes: Values are expressed in mean \pm standard deviation (SD).

Abbreviations: $\mathrm{Cl}$, confidence interval; CSI, Central Sensitization Inventory; EPDS, Edinburgh Postnatal Depression Scale; HADS, Hospital Anxiety and Depression Scale; OR, odds ratio; PCS, Pain Catastrophizing Scale; PND, postnatal depression.

development, ${ }^{9}$ we investigated if these questions could also be applied in identifying those associated with developing PND. ${ }^{13}$ In this study, anxiety about upcoming surgery was an independent association factor with PND at 6

Table 4 Multivariate Logistics Regression for Risk Factors for PND

\begin{tabular}{|l|l|l|}
\hline Risk Factors $^{\mathbf{a}}$ & $\begin{array}{l}\text { Adjusted OR } \\
\mathbf{9 5 \%} \mathbf{~ C I})\end{array}$ & p-value \\
\hline Pre-operative pain score (with & $1.65(1.12,2.44)$ & 0.0110 \\
movement) & $1.02(1.00,1.04)$ & 0.0429 \\
Anxiety about upcoming surgery & $0.48(0.29,0.79)$ & 0.0038 \\
Anticipated pain medication needs & $1.05(1.01,1.09)$ & 0.0156 \\
Pre-operative CSI & $1.25(1.07,1.45)$ & 0.0041 \\
Pre-operative HADS-anxiety &
\end{tabular}

Notes: ${ }^{a}$ Adjusted ORs were obtained from multivariate logistic regression by taking potential confounders $(p<0.05)$ identified by univariate analysis.

Abbreviations: $\mathrm{Cl}$, confidence interval; CSI, Central Sensitization Inventory; HADS, Hospital Anxiety and Depression Scale; OR, odds ratio; PND, postnatal depression. to 10 weeks post-Cesarean delivery. Surprisingly, we found a reverse relationship between anticipated pain medication needs and the development of PND. This finding reflects the complexity of the post-Cesarean delivery experience. Our further analysis did not show any significant association between anticipated pain medication needs and other risk factors including childbirth experiences (past Cesarean delivery, number of children) and 24 and $48 \mathrm{hrs}$ post-Cesarean pain scores. We suggest that one possible explanation is that individuals with perceived greater pain medication needs could be more open to acknowledge and receive treatment. Hence, those with lower perceived need for pain medication may be less willing to acknowledge depressive symptoms. Previous study demonstrated that almost $80 \%$ of patients with alcohol, drug, and mental (ADM) disorders with unmet clinical needs (defined as 1-year disorder with no ADM treatment) perceived no need for treatment, and that advanced age and lower educational level was associated with this low- 


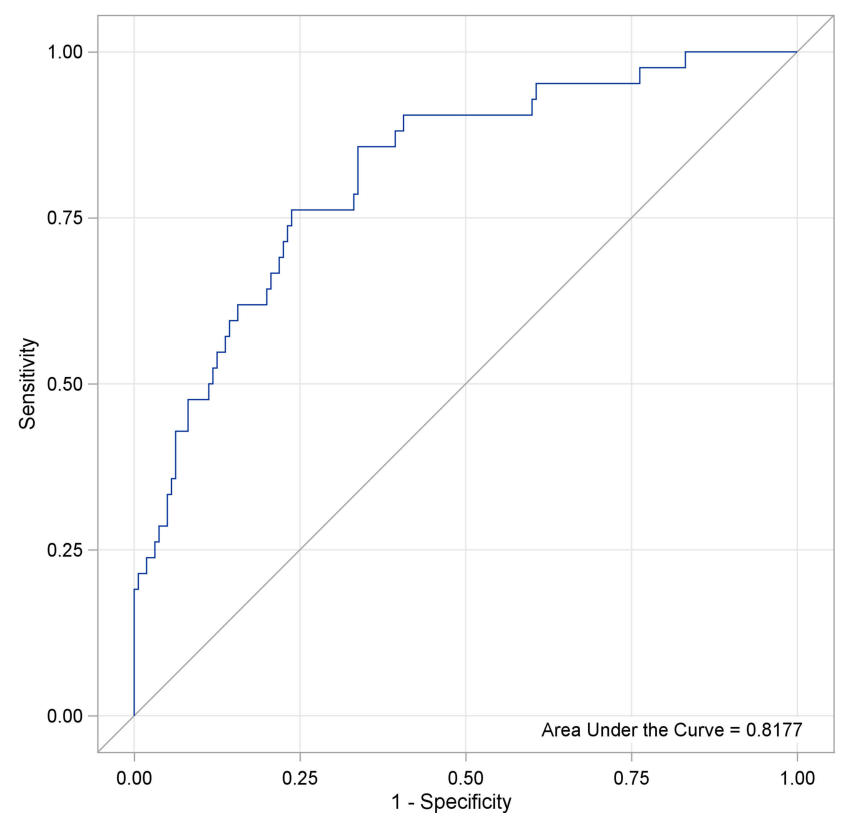

Figure 3 Receiver operating characteristic (ROC) of the suggested multivariate model.

perceived need. ${ }^{25}$ We also acknowledge the possibilities that variances in other physical awareness and societal influences, as well as past experiences, could further affect this hypothesis. ${ }^{26}$ More work will need to be done to explore these possible mechanisms.

This study used the anxiety symptom screening tool, HADS, to screen anxiety disorders during pre- and postCesarean period to reflect the changes in anxiety levels during hospital stay and at 6 to 10 weeks post-Cesarean delivery. The nature of this scale does not only cover the anxiety symptoms but also on common depressive symptomatology, and has been demonstrated to be well correlated with EPDS in pregnant patients (Spearman's rank correlation coefficient $=0.74, \mathrm{p}<0.05) .{ }^{27}$ These are in line with previous findings that anxiety symptoms are present throughout the perinatal period. ${ }^{28}$ Apart from the general anxiety as measured via HADS, we also assessed patients' anxiety about upcoming surgery which is found to be an independent association factor of PND in our study. By examining the course of anxiety levels on the day of the Cesarean delivery, Hepp et al demonstrated that subjective anxiety reached at peak values before Cesarean delivery, and significantly decreased from admission to skin suturing. Our findings on the positive association between anxiety about upcoming surgery and PND are suggestive that these simple questions on patients' perception and anticipation may be useful in predicting PND in patients undergoing Cesarean delivery. ${ }^{29}$

Central sensitization refers to the central amplification of pain, and is characterized by allodynia, hyperalgesia, expansion of the pain field and prolonged pain after stimulus removal. ${ }^{17}$ Several studies reported that depression and anxiety are closely associated with central sensitization, ${ }^{30,31}$ however the relationship of the latter with PND in Cesarean delivery is lacking. To our knowledge, this is the first study that utilized CSI to evaluate central sensitization in patients undergoing Cesarean delivery, demonstrating a positive association between higher CSI score and PND. Dysfunctional hypothalamo-pituitaryadrenal axis and disturbed monoamine transmission were suggested to be the causal link between central sensitization and the co-morbidity of pain and depression. ${ }^{32}$ However, the observations were mainly conducted in patients with chronic fatigue syndrome and fibromyalgia, ${ }^{33}$ both of which are characterized very differently from the pregnancy associated with psychological and physiological changes.

Previous studies have reported Cesarean delivery as a risk factor for PND, ${ }^{10,11}$ however limited evidence is available on the pre-operative factors during Cesarean delivery that is associated with PND. ${ }^{9}$ To our best knowledge, this is the first study looking into analgesic and psychological factors to generate an association model in PND after Cesarean delivery. There are several limitations in this study. The study was conducted in a study population with Chinese being the major ethnicity, and hence may not provide wider spectrum of information in other ethnicities. Other confounding factors such as the history of depression and mental illness, intraoperative and postoperative factors (endocrine hormone levels, infection, fever, etc.) as well as socioeconomic factors (education, income, occupation) were not collected that could be used for adjustments. We did not determine whether preexisting pain, not due to any disease, was present in patients before the delivery, and it is conceivable that patients classified in this study as PND could have prior pain problems and a heightened fear-avoidance status of childbirth and surgery. The findings reported herein are limited only to women undergoing elective Cesarean delivery in our institution. Patients who went for emergency Cesarean delivery may have a higher prevalence of PND as compared with elective Cesarean delivery, ${ }^{34}$ as the psychological and pain profile before delivery would be different from the present study. Finally, we utilized EPDS 
scores to assess the occurrence of PND; it is notable that EPDS is not a diagnostic instrument, but rather a screening tool to detect PND, and a positive predictive value may reflect transient mood lability, instead of depressive symptoms for PND. ${ }^{19}$ The use of different cut-off for EPDS score may be context- and condition-specific, with most of the studies reporting a use of scoring of 12 or 13 for major depression, or a cut-off score of 9 or 10 in minor depressed conditions. $^{35}$ While we acknowledge that the use of a different cut-off may yield different outcomes, it is notable that the Asian population tend to underreport their mood symptoms due to negative judgments and stigmatization, and hence the use of a slightly lower criteria may increase sensitivity of the analysis by including more cases of minor depression. ${ }^{36,37}$

\section{Conclusion}

In this study, increased pre-operative central sensitization, anxiety about upcoming surgery, and pre-operative HADS anxiety were associated with increased PND at 6 to 10 weeks post-Cesarean delivery. We also found that increased preoperative pain score with movement and lower anticipated pain medication needs were also independent association factors for the development of PND. The ROC analysis provided evidence that a combination of pain and psychological factors contribute in the association of PND development. Therefore, the need to focus on perioperative pain management and patients' expectations for Cesarean delivery to reduce longer-term PND development warrants further investigation. Prospective studies to validate our findings will be needed to confirm the impact of these predictive factors in the development of PND. Future research also evaluate the usefulness of this model to be implemented in perioperative screening to allow risk stratification and early intervention to reduce PND after Cesarean delivery.

\section{Abbreviations}

ADM, alcohol, drug, and mental; ASA, American Society of Anesthesiologists; BMI, body mass index; CI, confidence interval; CSI, Central Sensitization Inventory; EPDS, Edinburgh Postnatal Depression Scale; HADS, Hospital Anxiety and Depression Scale; IQR, interquartile range; LA, local anesthetic; MTS, mechanical temporal summation; OR, odds ratio; NRS, Numerical Rating Scale; PCS, Pain Catastrophizing Scale; PND, postnatal depression; ROC, Receiver operating characteristic; SD, standard deviation; STROBE, Strengthening the Reporting of Observational studies in Epidemiology.

\section{Data Sharing Statement}

The datasets generated and analyzed in this work are available for anyone who wishes to access the data by contacting the corresponding author.

\section{Ethics and Consent Statement}

The study was approved by the SingHealth Centralized Institutional Review Board, Singapore (SingHealth CIRB Ref: 2017/2381), and registered on Clinicaltrials.gov (NCT03645239). The authors declare that all the recruited patients provided informed consent, and that this work was conducted in accordance with the Declaration of Helsinki.

\section{Consent}

All patients provided informed consent on the use of their de-identified data for publication purpose.

\section{Acknowledgments}

We would like to thank Ms Juan Liu (Senior Clinical Research Coordinator) and Ms Agnes Teo (Senior Clinical Research Coordinator) for their administrative support in this work.

\section{Author Contributions}

All authors contributed to the study design, data analysis and discussion, drafting and/or revising the manuscript. All authors approved the final version of the manuscript, and agree to be accountable for all aspects of this work.

\section{Funding}

This work was supported by the funding from the SingHealth Duke-NUS Academic Medical Centre, Anesthesiology and Perioperative Science Academic Clinical Program Pilot Research Grant (Grant no. ANAESPRG18/02). The aforementioned sponsor was not involved in the study activities.

\section{Disclosure}

All authors report no conflicts of interest in this work.

\section{References}

1. McCoy S, Beal JM, Saunders B, Hill EN, Payton ME, Watson GH Risk factors for postpartum depression: a retrospective investigation. J Reprod Med. 2008;53(3):166-170.

2. Sword W, Kurtz Landy C, Thabane L, et al. Is mode of delivery associated with postpartum depression at 6 weeks: a prospective cohort study. BJOG. 2011;118(8):966-977. doi:10.1111/bjo.2011.11 8.issue-8 
3. Gelaye B, Rondon MB, Araya R, Williams MA. Epidemiology of maternal depression, risk factors, and child outcomes in low-income and middle-income countries. Lancet Psychiatry. 2016;3(10):973-9 82. doi:10.1016/S2215-0366(16)30284-X

4. Boerma T, Ronsmans C, Melesse DY, et al. Global epidemiology of use of and disparities in caesarean sections. Lancet. 2018;392 (10155):1341-1348. doi:10.1016/S0140-6736(18)31928-7

5. Sun K, Pan P. Persistent pain after cesarean delivery. Int $J$ Obstet Anesth. 2019;40:78-90. doi:10.1016/j.ijoa.2019.06.003

6. Bair MJ, Robinson RL, Katon W, Kroenke K. Depression and pain comorbidity: a literature review. Arch Intern Med. 2003;163 (20):2433-2445. doi:10.1001/archinte.163.20.2433

7. Williams L. Prevalence and impact of depression and pain in neurology outpatients. J Neurol Neurosurg Psychiatry. 2003;74(11):15 87-1589. doi:10.1136/jnnp.74.11.1587

8. Fishbain DA, Cutler R, Rosomoff HL, Rosomoff RS. Chronic pain-associated depression: antecedent or consequence of chronic pain? A review. Clin J Pain. 1997;13(2):116-137. doi:10.1097/000 02508-199706000-00006

9. Eisenach JC, Pan PH, Smiley R, Lavand'homme P, Landau R, Houle TT. Severity of acute pain after childbirth, but not type of delivery, predicts persistent pain and postpartum depression. Pain. 2008;140(1):87-94. doi:10.1016/j.pain.2008.07.011

10. Boyce PM, Todd AL. Increased risk of postnatal depression after emergency caesarean section. Med J Aust. 1992;157(3):172-174. doi:10.5694/mja2.1992.157.issue-3

11. Xu H, Ding Y, Ma Y, Xin X, Zhang D. Cesarean section and risk of postpartum depression: A meta-analysis. $J$ Psychosom Res. 2017;97:118-126. doi:10.1016/j.jpsychores.2017.04.016

12. Tonei V. Mother's mental health after childbirth: does the delivery method matter? J Health Econ. 2019;63:182-196. doi:10.1016/j. jhealeco.2018.11.006

13. Pan PH, Tonidandel AM, Aschenbrenner CA, Houle TT, Harris LC, Eisenach JC. Predicting acute pain after cesarean delivery using three simple questions. Anesthesiology. 2013;118(5):1170-1179. doi:10.10 97/ALN.0b013e31828e156f

14. Carter FA, Frampton CM, Mulder RT. Cesarean section and postpartum depression: a review of the evidence examining the link. Psychosom Med. 2006;68(2):321-330. doi:10.1097/01.psy.00002047 $87.83768 .0 \mathrm{c}$

15. Weissman-fogel I, Granovsky Y, Crispel Y, et al. Enhanced presurgical pain temporal summation response predicts post-thoracotomy pain intensity during the acute postoperative phase. J Pain. 2009;10 (6):628-636. doi:10.1016/j.jpain.2008.12.009

16. Sullivan MJ, Bishop SR, Pivik J. The pain catastrophizing scale: development and validation. Psychol Assess. 1995;7(4):524. doi:10. 1037/1040-3590.7.4.524

17. Mayer TG, Neblett R, Cohen H, et al. The development and psychometric validation of the central sensitization inventory. Pain Pract. 2012;12(4):276-285. doi:10.1111/ppr.2012.12.issue-4

18. Zigmond AS, Snaith RP. The hospital anxiety and depression scale. Acta Psychiatr Scand. 1983;67(6):361-370. doi:10.1111/acp.1983.67.issue-6

19. Cox J, Holden J. Perinatal mental health: A guide to the Edinburgh Postnatal Depression Scale (EPDS). Royal College of Psychiatrists; 2003.

20. Orbach-zinger S, Aviram A, Fireman S, et al. Severe pain during local infiltration for spinal anaesthesia predicts post-caesarean pain. Eur J Pain. 2015;19(9):1382-1388. doi:10.1002/ejp.737

21. Chandrasekaran N, De Souza LR, Urquia ML, et al. Is anemia an independent risk factor for postpartum depression in women who have a cesarean section?-A prospective observational study. BMC Pregnancy Childbirth. 2018;18(1):400. doi:10.1186/s12884-018-2032-6
22. Ecker J. Elective cesarean delivery on maternal request. JAMA. 2013;309(18):1930-1936. doi:10.1001/jama.2013.3982

23. Bennett IM, Coco A, Coyne JC, et al. Efficiency of a two-item pre-screen to reduce the burden of depression screening in pregnancy and postpartum: an IMPLICIT network study. J Am Board Fam Med. 2008;21(4):317-325. doi:10.3122/jabfm.2008.04.080048

24. Limlomwongse N, Liabsuetrakul T. Cohort study of depressive moods in Thai women during late pregnancy and 6-8 weeks of postpartum using the Edinburgh Postnatal Depression Scale (EPDS). Archiv women's mental health. 2006;9(3):131-138. doi:10. 1007/s00737-005-0115-7

25. Edlund MJ, Unützer J, Curran GM. Perceived need for alcohol, drug, and mental health treatment. Soc Psychiatry Psychiatr Epidemiol. 2006;41(6):480-487. doi:10.1007/s00127-006-0047-1

26. Haugland S, Wold B. Subjective health complaints in adolescencereliability and validity of survey methods. $J$ Adolesc. 2001;24 (5):611-624. doi:10.1006/jado.2000.0393

27. Adouard F, Glangeaud-freudenthal N, Golse B. Validation of the Edinburgh postnatal depression scale (EPDS) in a sample of women with high-risk pregnancies in France. Archiv women's mental health. 2005;8(2):89-95. doi:10.1007/s00737-005-0077-9

28. Kuo S-Y, Chen S-R, Tzeng Y-L. Depression and anxiety trajectories among women who undergo an elective cesarean section. PLoS One. 2014;9(1):e86653. doi:10.1371/journal.pone.0086653

29. Hepp P, Hagenbeck C, Burghardt B, et al. Measuring the course of anxiety in women giving birth by caesarean section: a prospective study. BMC Pregnancy Childbirth. 2016;16(1):113. doi:10.1186/ s12884-016-0906-z

30. Hansson P, Backonja M, Bouhassira D. Usefulness and limitations of quantitative sensory testing: clinical and research application in neuropathic pain states. Pain. 2007;129(3):256-259. doi:10.1016/j. pain.2007.03.030

31. Eisenach J, Coghill R, Pan P, Harris L. Predictors of pain and analgesic use after cesarean section. Anesthesiology. 2005; 102 (Suppl 1):A-10.

32. Blackburn-munro G. Hypothalamo-pituitary-adrenal axis dysfunction as a contributory factor to chronic pain and depression. Curr Pain Headache Rep. 2004;8(2):116-124. doi:10.1007/s11916-004-0025-9

33. Price DD, Staud R, Robinson ME, Mauderli AP, Cannon R, Vierck CJ. Enhanced temporal summation of second pain and its central modulation in fibromyalgia patients. Pain. 2002;99(1-2):4959. doi:10.1016/S0304-3959(02)00053-2

34. Meky HK, Shaaban MM, Ahmed MR, Mohammed TY. Prevalence of postpartum depression regarding mode of delivery: a cross-sectional study. J Matern Fetal Neonatal Med. 2019;1-8. doi:10.1080/1476 7058.2019 .1571572

35. Gibson J, McKenzie-McHarg K, Shakespeare J, Price J, Gray R. A systematic review of studies validating the Edinburgh Postnatal Depression Scale in antepartum and postpartum women. Acta Psychiatr Scand. 2009;119(5):350-364. doi:10.1111/j.1600-0447.20 09.01363.x

36. Georg Hsu L, Wan YM, Chang H, Summergrad P, Tsang BY, Chen H. Stigma of depression is more severe in Chinese Americans than Caucasian Americans. Psychiatry. 2008;71(3):210-218. doi:10. 1521/psyc.2008.71.3.210

37. Wynaden D, Chapman R, Orb A, McGowan S, Zeeman Z, Yeak S. Factors that influence Asian communities' access to mental health care. Int J Ment Health Nurs. 2005;14(2):88-95. doi:10.1111/inm.20 05.14.issue-2 


\section{Publish your work in this journal}

Neuropsychiatric Disease and Treatment is an international, peerreviewed journal of clinical therapeutics and pharmacology focusing on concise rapid reporting of clinical or pre-clinical studies on a range of neuropsychiatric and neurological disorders. This journal is indexed on PubMed Central, the 'PsycINFO' database and CAS, and is the official journal of The International Neuropsychiatric Association (INA). The manuscript management system is completely online and includes a very quick and fair peer-review system, which is all easy to use. Visit http://www.dovepress.com/testimonials.php to read real quotes from published authors.

Submit your manuscript here: https://www.dovepress.com/neuropsychiatric-disease-and-treatment-journal 